\title{
GENERAL RELATIVISTIC EFFECTS ON THE OHMIC DECAY OF CRUSTAL MAGNETIC FIELDS IN NEUTRON STARS
}

\author{
SUJAN SENGUPTA \\ Indian Institute of Astrophysics \\ Koramangala, Bangalore 560 034, India \\ sujan@iiap.ernet.in
}

Received : 17 December 1996; accepted : 29 January 1997

To appear in the Astrophysical Journal Letters 


\begin{abstract}
The ohmic decay of magnetic fields confined within the crust of neutron stars is considered by incorporating the effect of space-time curvature produced by the intense gravitational field of the star. It is shown that general relativistic effect reduces the magnetic field decay rate substaintially and specially at the late time of the evolution the decay rate decreases by several orders of magnitude when compared with the case without the relativistic effect.
\end{abstract}

Subject headings: magnetic fields — relativity — stars : neutron 


\section{INTRODUCTION}

The magnetic field evolution in neutron stars has been a subject of much discussion over the years both in the observational and in the theoretical context. Calculations of ohmic decay of dipolar magnetic fields were performed by Sang \& Chanmugam (1987) who demonstrated that the field does not decay exponentially. The reviews by Lamb (1991), Chanmugam (1992) and Phinney \& Kulkarni (1994) provide the present understanding on the decay of magnetic fields in isolated neutron stars. Haensel, Urpin \& Yakovlev (1990) pointed out to the possibility that magnetic fields in the core could decay rapidly by ambipolar diffusion. The studies of magnetic field configurations in which the field vanishes in the stellar core (Sang \& Chanmugam 1987; Chanmugam \& Sang 1989; Urpin \& Muslimov 1992; Urpin \& Van Riper 1993) show that because of the relatively low electrical conductivity of crustal matter, the decay times may be short enough to be of observational interest if the impurity concentration is high and the field is initially confined to a small part of the crust. On the other hand, considering field configurations that do not vanish in the core, Pethick \& Sahrling (1995) showed that the shortest possible decay time is about two orders of magnitude longer than the characteristic time-scale for decay of configurations in which the magnetic field vanishes in the core.

In all the investigations, an important feature of neutron stars, the space-time curvature produced by the intense gravitational field has not been taken into account although it is well-known that the space-time curvature exterior and interior to neutron stars can significantly alter the electromagnetic field (Wasserman \& Shapiro 1983; Sengupta 1995; Bocquet, et al. 1995).

In this letter it is demonstrated, by adopting a simplified model, that the decay rate decreases significantly when the general relativistic effect is taken into consideration. It is, however, worth mentioning that due to lack of proper understanding on the initial 
configuration of the magnetic field and of the impurity parameter which plays crucial role in determining the electrical conductivity, no model presented so far can be attributed to represent the quantitative feature of the actual situation. Nevertheless, all these investigations are important from the qualitative point of view and provide significant insight on the decay of the magnetic field. The scope of the present work although is also limited to an idealistic situation but it is sufficient to demonstrate the important role played by curved space-time at the crust of neutron stars in governing the decay of magnetic fields. The present result indicates that the space-time curvature produced by the intense gravitational field at the crust can lead to characteristic decay-times much longer than the existing estimations.

\section{MAGNETIC FIELD EVOLUTION IN CURVED SPACE-TIME}

If hydrodynamic motions are negligible and the anisotropy of the electrical conductivity is small, the induction equation in flat space-time can be written as:

$$
\frac{\partial \mathbf{B}}{\partial t}=-\nabla \times\left(\frac{c^{2}}{4 \pi \sigma} \nabla \times B\right)
$$

where $\sigma$ is the electrical conductivity.

If a stationary gravitational field is taken into account, the corresponding induction equation in curved space-time can be written as:

$$
\frac{\partial F_{k j}}{c \partial t}=\frac{c}{4 \pi}\left[\frac{\partial}{\partial x^{k}}\left\{\frac{1}{\sqrt{-g}} \frac{1}{\sigma u^{0}} g_{i j} \frac{\partial}{\partial x^{l}}\left(\sqrt{-g} F^{i l}\right)\right\}-\frac{\partial}{\partial x^{j}}\left\{\frac{1}{\sqrt{-g}} \frac{1}{\sigma u^{0}} g_{i k} \frac{\partial}{\partial x^{l}}\left(\sqrt{-g} F^{i l}\right)\right\}\right],
$$

where $F_{i j}$ is the electromagnetic field tensor, $g_{i j}$ is the components of space-time metric that describes the background geometry, $g=\operatorname{det}\left|g_{i j}\right|$ and $u^{0}$ is the time component of the velocity vector of the fluid (I have considered $u^{i}=0$ ). Here and afterwards latin indices run over spatial co-ordinates only whereas greek indices run over both time and space co-ordinates. 
Now we need to choose a space-time metric which describes the geometry of the region under consideration. Although, in Newtonian theory the gravitational field of a rotating body is the same as that of a non-rotating body, in general relativity rotation affects the space-time geometry. However, in the absence of any suitable metric that can describe the space-time geometry inside a rotating neutron star, I consider, in the present investigation, a stationary and static gravitational field. Further, it is well-known (see for example Datta, Thampan \& Bhattacharya 1995 and references therein) that the gravitational mass of the entire crust of neutron stars consists of less than $3 \%$ of the total mass of the star for any equation of state and hence the self gravitation of the crust is negligible compared to the gravitational field due to the core. Since in the present work, the magnetic field is considered to be confined within the outermost crust and the magnetic field strength therein is supposed to be sufficiently low compared to the gravitational field so that the space-time curvature is not affected by the electromagnetic field, one can very well adopt the exterior Schwarzschild metric which on the other hand simplifies the calculations. The metric is given by:

$$
d s^{2}=\left(1-\frac{2 m}{r}\right) c^{2} d t^{2}-\left(1-\frac{2 m}{r}\right)^{-1} d r^{2}-r^{2}\left(d \theta^{2}+\sin ^{2} \theta d \phi^{2}\right),
$$

where $m=M G / c^{2}, M$ being the total gravitational mass of the core. Since, the crust consists of less than a few percent of the total gravitational mass, $M$ can be regarded as the total mass of the star.

If $F_{(\alpha \beta)}$ are the components of the electromagnetic field tensor in a local Lorentz frame, then the components of the electromagnetic field tensor $F_{\gamma \delta}$ are defined in the curved space-time through the relation:

$$
F_{(\alpha \beta)}=\lambda_{(\alpha)}^{\gamma} \lambda_{(\beta)}^{\delta} F_{\gamma \delta},
$$

where $\lambda_{(\beta)}^{\alpha}$ are the non-zero components of the orthonormal tetrad of the local Lorentz frame for the Schwarzschild geometry given in Sengupta (1995). 
Following the convention, I restrict myself by the consideration of the decay of a dipolar magnetic field which has axial symmetry so that the vector potential $\mathbf{A}$ may be written as $\left(0,0, A_{\phi}\right)$ in spherical polar co-ordinates where $A_{\phi}=A(r, \theta, t)$. Since the hydrodynamic motion is negligible so $u^{i}=d x^{i} / d s=0$ and the metric gives $u^{0}=(1-2 m / r)^{-1 / 2}$.

Substituting the metric components in equation (2) and using the definition $F_{\alpha \beta}=\left(A_{\beta, \alpha}-A_{\alpha, \beta}\right)$, one obtains the induction equation in Schwarzschild geometry in term of the vector potential as:

$$
\frac{\partial A_{\phi}}{\partial t}=\frac{c^{2}}{4 \pi \sigma}\left(1-\frac{2 m}{r}\right)^{1 / 2} \sin \theta\left[\frac{\partial}{\partial r}\left\{\left(1-\frac{2 m}{r}\right) \frac{1}{\sin \theta} \frac{\partial A_{\phi}}{\partial r}\right\}+\frac{\partial}{\partial \theta}\left(\frac{1}{r^{2} \sin \theta} \frac{\partial A_{\phi}}{\partial \theta}\right)\right] .
$$

Now for the flat space-time I choose $A_{\phi}=f(r, t) \sin \theta / r$ where $r$ and $\theta$ are the spherical radius and polar angle respectively and one gets from equation (11)

$$
\frac{\partial^{2} f(x, t)}{\partial x^{2}}-\frac{2}{x^{2}} f(x, t)=\frac{4 \pi R^{2} \sigma}{c^{2}} \frac{\partial f(x, t)}{\partial t}
$$

where $x=r / R$ and $R$ is the radius of the star.

For the general relativistic case, the choice is guided by the form of the time-independent dipole magnetic field in Schwarzschild geometry obtained by Wasserman and Shapiro (1983) and can be written as $A_{\phi}=-g(x, t) \sin ^{2} \theta$. Hence, from equation (5) one obtains

$$
\left(1-\frac{y}{x}\right)^{1 / 2}\left[\left(1-\frac{y}{x}\right) \frac{\partial^{2} g(x, t)}{\partial x^{2}}+\frac{y}{x^{2}} \frac{\partial g(x, t)}{\partial x}-\frac{2}{x^{2}} g(x, t)\right]=\frac{4 \pi R^{2} \sigma}{c^{2}} \frac{\partial g(x, t)}{\partial t}
$$

where $y=2 m / R$

For both the cases, I impose the usual boundary conditions which are (i) in the deep layer of the crust, the magnetic field vanishes and (ii) at the outer boundary of the crust, the field matches onto an exterior dipole magnetic field. 


\section{THE MODEL}

I shall follow Urpin \& Van Riper (1993) in my approach with one exception. To simplify the calculations, I neglect the neutron star cooling which can significantly decrease the field decay rate since the conductivity of the crust depends on the temperature $T$. The qualitative nature of the present results, however, will not be altered if one incorporates the neutron star cooling as long as $\sigma$ itself is independent of the space-time curvature. Calculations of ohmic decay of bipolar magnetic field without the effect of the neutron star cooling was performed by Sang \& Chanmugam (1987). Their results have been used to check the numerical accuracy of the present results corresponding to flat space-time.

As mentioned in the introduction, any given magnetic field configuration in flat space-time would be modified by the curvature of space-time produced by the gravitational field. If initially (at $t=0$ )

$$
A_{\phi}(r, \theta, 0)=A_{\phi}(r, \theta)=\frac{f(r, 0)}{r} \sin \theta=\frac{f(r)}{r} \sin \theta
$$

for flat space-time, then for curved space-time

$$
A_{\phi}(r, \theta, 0)=A_{\phi}(r, \theta)=-\frac{3 r f(r)}{8 m^{3}} s(r) \sin ^{2} \theta
$$

where $s(r)$ is the general relativistic correction factor. Hence, it can be shown (Wasserman \& Shapiro 1983) that if one assumes the initial value of $f(r, t)=f(r)$ for flat space-time, then for curved space-time

$$
g(r, 0)=g(r)=\frac{3 r f(r)}{8 m^{3}}\left[r^{2} \ln \left(1-\frac{2 m}{r}\right)+2 m r+2 m^{2}\right]
$$

Clearly, if $r \rightarrow \infty, g(r) \rightarrow f(r)$.

I have considered the decay of the magnetic field which initially occupies the surface layers of the crust upto a depth $x=0.955$ which corresponds to the density $5 \times 10^{11} \mathrm{gcm}^{-3}$. 
Following the approaches of Urpin and Van Riper (1993) I have calculated the electrical conductivity within the crust that has been derived by Urpin \& Yakovlev (1980). The effect of electron-ion scattering has been neglected since the region where this effect could be important is sufficiently thin. The impurity parameter $Q$ has been taken as 0.001 and the conductivity is calculated by assuming the region under consideration to be isothermal with a constant temperature $T=10^{7} \mathrm{~K}$. Results with $T=10^{5} \mathrm{~K}$ have also been presented for few relevant cases. The calculations have been performed by considering a neutron star of mass $1.4 M_{\odot}$ and radius $10.6 \mathrm{~km}$.

\section{RESULTS AND DISCUSSIONS}

Equations (6) and (7), with the corresponding boundary conditions, have been solved numerically. The calculations have been performed by making use of the standard CrankNicholson differencing scheme. For the sake of comparison of the results, the magnetic field in Schwarzschild geometry has been transformed into a local Lorentz frame by using equation (4). If one takes the mass $M$ sufficiently low $\left(<0.01 M_{\odot}\right)$ then the decay profile calculated in the local Lorentz frame coincides with that for the flat space-time and for the present purpose this provides sufficient check for the numerical accuracy of the results. The effect of general relativity in governing the magnetic field decay is clearly depicted in the figures. Figure 1 shows the evolution of the surface magnetic field normalized to its initial value for both the general relativistic and non-relativistic cases. The qualitative nature of the decay profiles are same with that presented by Sang \& Chanmugam (1987) as in both the cases the effect of the neutron star cooling has not been taken into consideration. In the present work such type of effect is not relevant since it is expected that the cooling process and hence the electrical conductivity are independent of the space-time curvature.

EDITOR: PLACE FIGURE 1 HERE. 
In Figure 1 the decay of the surface magnetic field is presented for neutron stars with masses $1 M_{\odot}, 1.4 M_{\odot}$ and $1.8 M_{\odot}$ and with the same radius $R=10.6 \mathrm{~km}$. However, the electrical conductivity is calculated by adopting $1.4 M_{\odot}$ mass configuration. It is clear from the figure that the decay rate decreases significantly with the increase in mass, i.e., with the increase in the gravitational field for the whole period of evolution. Unlike the case for flat space-time, the decay rate gradually slows down as the time increases. In other words, the effect of general relativity becomes more significant with the increase in the age of the star. At the late stage of evolution, say, after 3 Gyr, the difference in the strength of the surface magnetic field between the two cases becomes as large as about 3 orders of magnitude. It is interesting to note that if the mass of the star is as high as $1.8 M_{\odot}$ which can be possible with any stiff equation of state of matter inside the star, then due to the effect of space-time curvature the decay rate decreases so dramatically that when the other physical effects such as cooling of the neutron star which too play significant role in reducing the decay rate, are incorporated the decay in the magnetic field could be too small to be determined observationally and during the whole life span of the star the strength of the magnetic field would remain almost the same with that of its initial value. Since general relativistic effect alone can reduce the decay rate significantly, observational evidence of faster decay rate may constrain the compactness of the neutron star and hence the equation of state of matter inside the star. It is worth mentioning here that the general relativistic effect on the magnetic field is less uncertain than any other physical phenomenon exterior or interior to isolated neutron stars.

\section{EDITOR: PLACE FIGURE 2 HERE.}

In Figure 2 the variation of the magnetic field along the radial points inside the crust at different times are presented for a neutron star of mass $1.4 M_{\odot}$. The initial field strength increases by the inclusion of general relativistic effect. This result is well known for 
time-independent dipole magnetic field (Wasserman \& Shapiro 1983; Sengupta 1995). After 5 Myr the strength of the magnetic field for the curved space-time becomes almost equal to the initial value of the field strength when the general relativistic effect is not included. Subsequently, for flat space-time the field strength at any time remains much lower than that for curved space-time. However, it cannot be inferred that due to the modification in the initial configuration of the magnetic field the final field strength becomes much higher for the general relativistic case than that for the case of flat space-time. Rather, the entire evolution should be modified by the general relativistic effect as indicated by equation (घ). As the temperature of the crust must decrease with time, the electrical conductivity becomes higher and hence at the late stage of evolution the decay rate should decrease substaintially irrespective of the nature of the surrounding space-time. In figure 2, the magnetic field strength at 1 Gyr with the crust temperature $T=10^{5} \mathrm{~K}$ for both the general relativistic and non-relativistic cases are presented. The results provide a confirmation of the above fact. Since the magnetic field strength for the general relativistic case is almost double to its value when relativistic effect is not considered, it is very much likely that the magnetic field decay should be too small to be detected observationally if both the cooling effect and the general relativistic effect are taken together into account. Therefore, detail investigations by considering the neutron star cooling and other physical phenomenon alongwith the general relativistic effect will be of much theoretical interest.

\section{CONCLUSIONS}

The important message which is conveyed by the present calculations is that whatever be the electrical conductivity of the crustal material, high or low, irrespective of the question whether the magnetic field vanishes at the core or not and whatever be the impurity

content of the neutron star crust, the decay time of the magnetic field is lengthened by the 
intense gravitational field that certainly exists inside the star. Sang \& Chanmugam (1987) showed that the decay is not exponential while Urpin \& Muslimov (1992) pointed out that even if the magnetic field is initially absent in the core, diffusion of the field into a highly conducting core would retard the surface field decay. Pethic and Sahrling (1995) suggested that if long decay times were established observationally, these could not necessarily imply as the evidence for matter in the stellar core having a high conductivity. Irrespective of all the uncertainties that still exist and require further theoretical investigations as well as observational evidences the present result can atleast provide a firm understanding that general relativity is certainly responsible if the decay time is indeed very long. Therefore, the present demonstration is important in the sense that it establishes a concrete restrictions on the theoretically possible ways of obtaining short ohmic decay times for magnetic fields in neutron stars. Further, the present results provide an interesting feature that the more is the compactness of the neutron star the longer is the decay times and hence the general relativistic effects on the decay of magnetic field could be a possible tool for restricting the equation of state of matter inside the star which determines the compactness of the neutron star.

The crucial lesson that the present results provide is that, in addition to the calculations of the conductivity with better estimation of the impurity content, the incorporation of the effect of superfluidity and superconductivity in the core and other physical effects such as Hall drift, full consideration of the general relativistic effects must be given in order to make more realistic estimates of decay times of the magnetic field.

I am grateful to late Professor N. C. Rana (1954-1996) who initiated this work and had been a constant source of encouragement till his sudden demise. Thanks are due to the anonymous referee for useful comments and constructive criticisms. 


\section{REFERENCES}

Bocquet, M., Bonazzola, S., Gourgoulhon, E., \& Novak, J. 1995, A\&A, 301, 757

Chanmugam, G. 1992, ARA\&A, 30,143

Chanmugam, G., \& Sang, Y. 1989, MNRAS, 241, 295

Datta, B., Thampan, A. V., \& Bhattacharya, D. 1995, J. Astrophys. Astron., 16, 375

Haensel, P., Urpin, V., \& Yakovlev, D. 1990, A\&A, 229, 133

Lamb, F. 1991, in ASP Conf. Proc. 20, Frontiers of Stellar Evolution, ed. D. Lambert (San Francisco: ASP), 299

Phinney, S., \& Kulkarni, S. 1994, ARA\&A, 32, 591

Pethic, C. J., \& Sahrling, M. 1995, ApJ, 453, L29

Sang, Y., \& Chanmugam, G. 1987, ApJ, 323, L61

Sengupta, S. 1995, ApJ, 449, 224

Urpin, V. A., \& Muslimov, A. G. 1992, MNRAS, 256, 261

Urpin, V. A., \& Van Riper, K. A. 1993, ApJ, 411, L87

Urpin, V. A., \& Yakovlev, D. G. 1980, Soviet Astron., 24, 303

Wasserman, I., \& Shapiro, S. L. 1983, ApJ, 265, 1036 


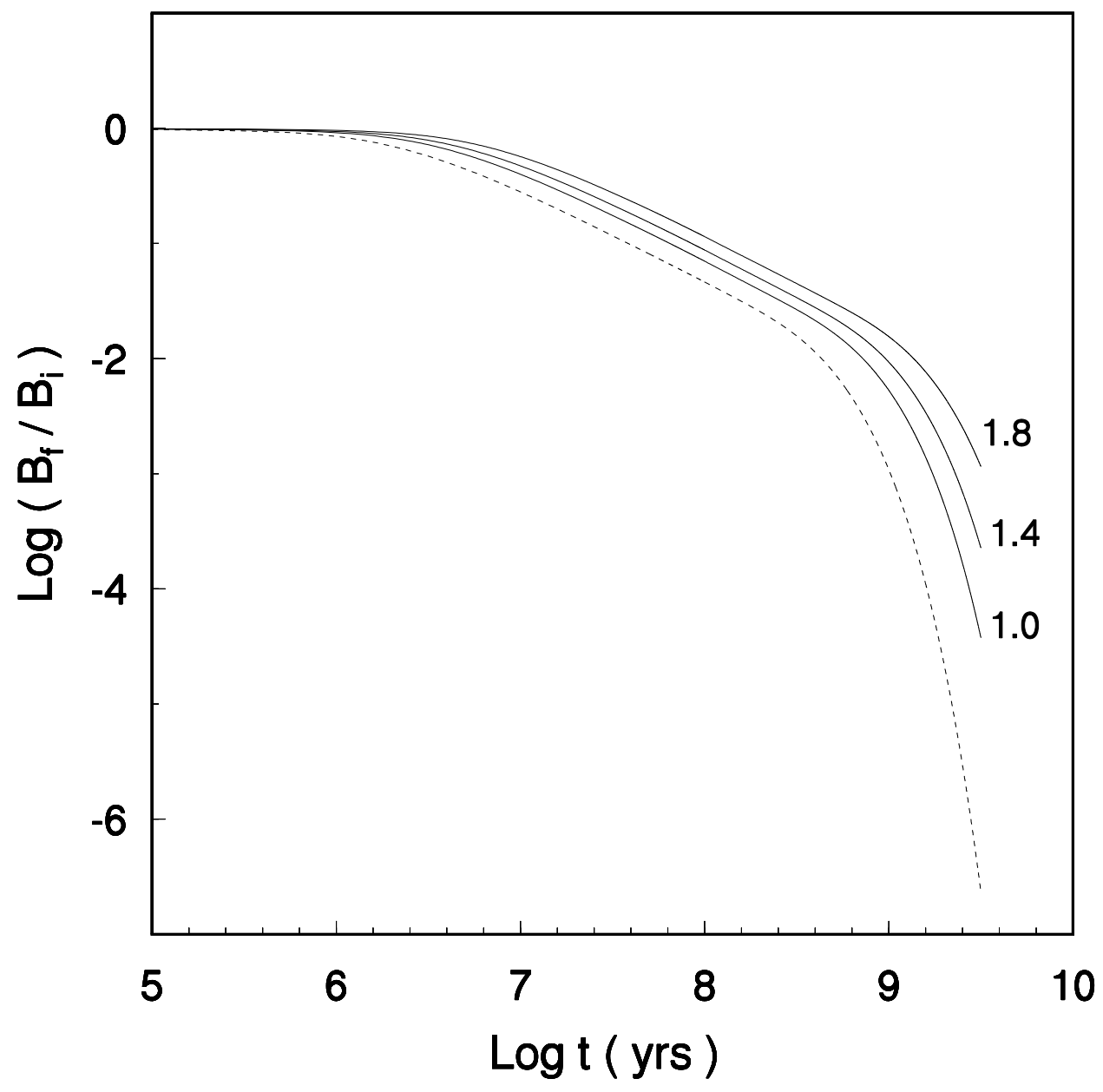

Fig. 1.- The evolution of surface magnetic field normalized to its initial value for flat and curved spacetimes. Solid lines represent the results for curved space-time while broken lines represent that for flat space-time. The numbers near the curves indicate mass in $1 M_{\odot}$. For all the cases the radius is taken as $R=10.6 \mathrm{~km}$ and the electrical conductivity is calculated for a $1.4 M_{\odot}$ neutron star crust. 


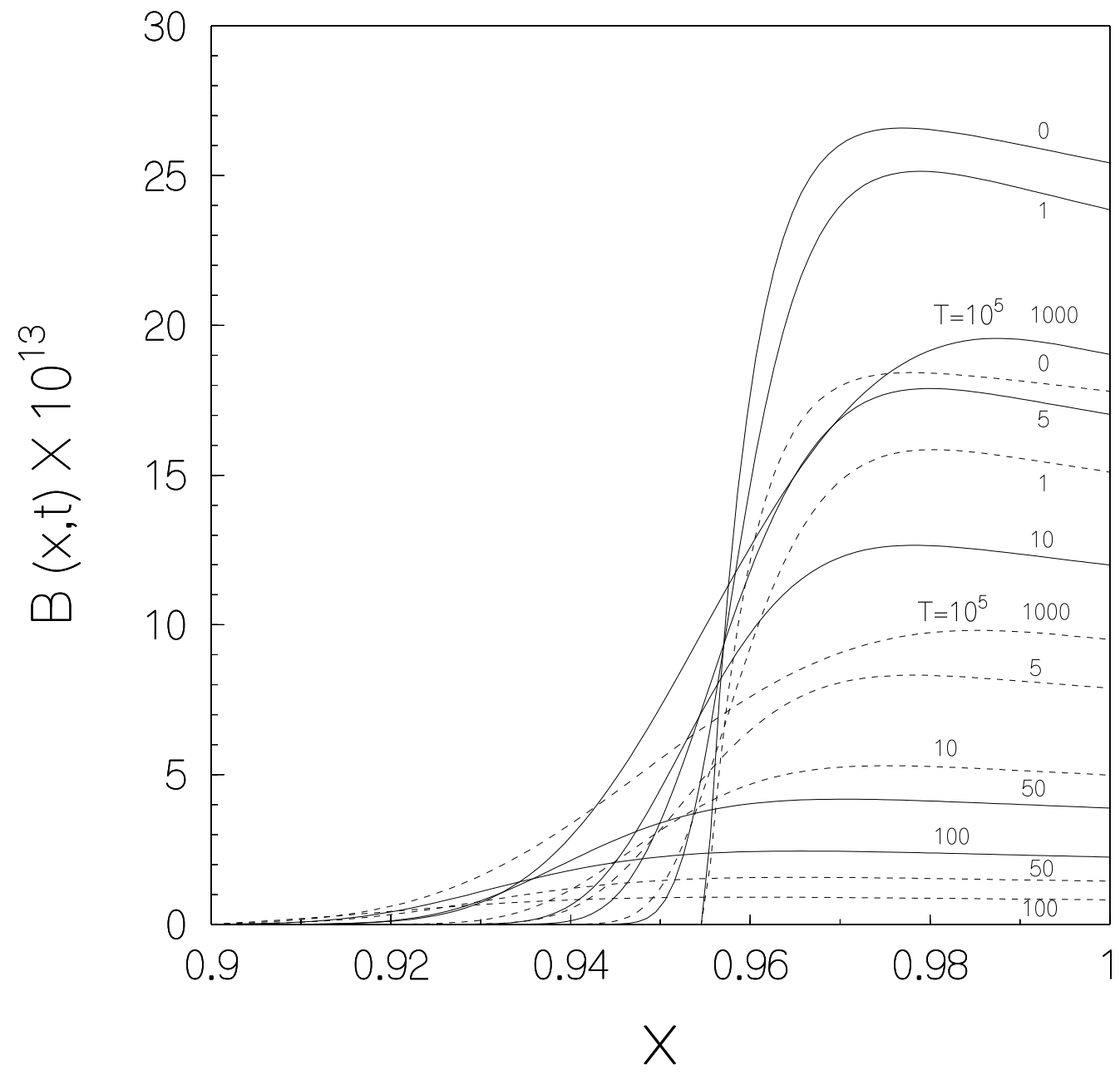

Fig. 2.- The dipole magnetic field at different time along the normalized radial points. The numbers near the curves indicate $t$ in Myr and the curves corresponding to $t=0$ indicate the initial value of the magnetic field. Unless indicated, all the curves present the results with temperature $T=10^{7} \mathrm{~K}$. Solid lines represent the case for curved space-time while broken lines represent that for flat space-time. 